Complications after surgical treatment of femoral neck fractures in men with alcohol dependence syndrome : retrospective register analysis of 154 cases

Kosola, Jussi

2017-07

Kosola , J , Kaipia , A , Laitinen , M K \& Nieminen , J 2017 , ' Complications after surgical treatment of femoral neck fractures in men with alcohol dependence syndrome :

retrospective register analysis of 154 cases ' , Archives of Orthopaedic and Trauma Surgery , vol. 137 , no. 7 , pp. 967-973 . https://doi.org/10.1007/s00402-017-2713-z

http://hdl.handle.net/10138/297840

https://doi.org/10.1007/s00402-017-2713-z

publishedVersion

Downloaded from Helda, University of Helsinki institutional repository.

This is an electronic reprint of the original article.

This reprint may differ from the original in pagination and typographic detail.

Please cite the original version. 


\title{
Complications after surgical treatment of femoral neck fractures in men with alcohol dependence syndrome: retrospective register analysis of 154 cases
}

\author{
Jussi Kosola $^{1} \cdot$ Antti Kaipia $^{2} \cdot$ Minna K. Laitinen $^{3,4}$ (D) Jyrki Nieminen ${ }^{3}$
}

Received: 18 January 2017/ Published online: 10 May 2017

(C) Springer-Verlag Berlin Heidelberg 2017

\begin{abstract}
Purpose One-third of hip fractures occur in men. The causes underlying hip fractures in men differ from those in women and include alcohol abuse. This retrospective register study evaluated the trends and results associated with different surgical treatment methods for nondisplaced and displaced femoral neck fractures in male patients with alcohol dependence syndrome.

Methods Men with hip fractures were identified from a local district hospital database. Alcohol dependence syndrome was identified as a diagnosis in medical records.

Results For displaced fractures, implant survival after total hip arthroplasty was significantly lower compared to hemiarthroplasty. For nondisplaced fractures, implant survival of cannulated screws was significantly lower compared to sliding hip screws. Overall patient survival for males with alcohol dependence syndrome with hip fracture was $62 \%$ at 1 year and $49 \%$ at 2 years. Patient survival in this population did not differ between displaced and nondisplaced fractures or among different surgical methods.

Conclusion Patients with alcoholism who had documented evidence of alcohol dependence syndrome represented
\end{abstract}

Minna K. Laitinen

minna.laitinen@fimnet.fi

1 Department of Orthopaedics and Traumatology, Helsinki University Hospital, Helsinki, Finland

2 Department of Surgery, Satakunta Central Hospital, Pori, Finland

3 Coxa Hospital for Joint Replacement, Tampere, Finland

4 Unit of Musculoskletal Surgery, Department of Orthopaedics, Tampere University Hospital, Pl 2000, 33521 Tampere, Finland nearly half of patients $<70$ years old with low-energy hip fracture. In patients with nondisplaced femoral neck fractures, stability of the internal fixation appeared to play a major role in implant survival; sliding hip screws should be considered over multiple cannulated screws. In patients with displaced fracture, total hip arthroplasty was associated with a significantly higher risk of complications leading to revision compared to hemiarthoplasty. Level of evidence Prognostic Level III.

Keywords Alcoholism - Femoral neck fracture . Arthroplasty · Hemiarthroplasty · Fracture fixation . Internal

\section{Introduction}

Hip fracture is a common fracture, particularly in older female patients. However, about one-third of hip fractures occur in men. The causes underlying osteoporosis and susceptibility to falling differ between women and men, as do mortality rates; male patients appear to have higher standardized mortality ratios compared to female patients [1].

Alcohol dependence syndrome (ADS) and smoking are known risk factors, especially among male patients [2, 3]. Alcoholics have an increased risk of developing osteopenia [4]; moreover, they can become increasingly uncoordinated, resulting in falls [5]. The association of hip fracture with alcohol consumption is nonlinear; light alcohol consumption is inversely associated with decreased hip fracture risk, whereas heavy alcohol consumption is associated with elevated hip fracture risk [6].

Optimal treatment of femoral neck fracture has been debated for decades and is especially controversy among 
alcoholics, as they are younger and have limited co-operation. The purpose of this retrospective register study was to evaluate the trends and results associated with different surgical methods to treat nondisplaced and displaced femoral neck fractures in male patients with ADS. Data were collected for the male Finnish population in the Pirkanmaa district region of Finland who were treated for hip fracture in 2002-2014.

\section{Materials and methods}

The Pirkanmaa district hospital area has a population of approximately 450,000 overall, of which 200,000 are men. Within the catchment area, four hospitals treat patients with hip fractures. The criteria for inclusion in this study were as follows: male patients $>20$ years old who had visited one of the four hospitals between 1 January 2002 and 31 December 2014 with a diagnosis of hip fracture as defined by ICD-10 codes S72.0, S72.1, and S72.2. Patients who were temporarily staying in Pirkanmaa but registered elsewhere were not included in this study. Eligible patients were identified by a computer-based search of electronic patient records and files both for proximal femoral fractures as well as for ADS (F10.2). All patient records were reviewed by all authors. We identified 1526 male patients with proximal femur fracture. Within this hip fracture population, 272 patients $(18 \%)$ were regular alcohol drinkers with documented evidence of having ADS and in 265 fractures, the energy was low (equal to fall from a standing height). 154 lowenergy fractures were localized to femoral neck and this patients' population was our main interest. Nondisplaced femoral neck fractures were treated with cannulated screws or SHS (use of cannulated screws or SHS was decided on a caseby-case basis) and displaced femoral neck fractures were treated with THA or HA. THA was selected for patients whose level of physical activity was higher and who were predicted to have a better overall outcome (Fig. 1).

Clinical data recorded from clinician notes included ADS as a diagnose, age, comorbidities, details of arthroplasty, and the Charlson age-adjusted comorbidity index (CCI) [7]. The primary outcome of the study was implant survival, with revision for any reason as an endpoint. Secondary outcomes were patient survival and number of revisions (e.g., for periprosthetic fracture, dislocation, and deep infection).

We derived the total number and age distribution of the population at risk ( $>20$ years old) for each year of the study period from the Statistics Finland website (http:// www.tilastokeskus.fi). Statistics Finland contains information about each registered inhabitant of Finland; extracting information about at-risk populations in any given region is simple. We then calculated the crude incidence rate using 10 -year intervals per 100,000 person-years.

\section{Statistical analysis}

We assessed implant survival by the Kaplan-Meier method with a log-rank test for univariate analysis. Cox regression analysis was used to identify independent factors that affected the CCI in primary complications. We used twosided Fisher's exact test to compare the study characteristics of each group. We used the Kruskal-Wallis test and the independent-samples median test to compare means and medians, respectively, for each variable. The endpoints survival and follow-up period were the first postoperative implant-related complication, death, or status on 5 May 2016. A $p$ value of $<0.05$ was considered statistically significant.

\section{Results}

Of the 154 femoral neck fracture patients, 42 (27\%) patients had their fracture treated by THA, 76 (49\%) with HA, $12(8 \%)$ with cannulated screws, and $24(16 \%)$ with SHS. THA was performed with different components that were selected on a case-by-case basis. By contrast, all patients who underwent HA received a cemented Lubinus monopolar implant (Link, Hamburg, Germany). The following ostesynthetic materials were used: ASNIS cannulated screws (Stryker Inc., Kalamazoo, MI, USA), dynamic hip screws (DHS; Synthes, West Chester, PA, USA), Gamma III nails (Stryker), and proximal femoral nails (PFN) as SHS (Synthes). The median age of the patients was 64 years (range 29-94 years). Median age was significantly lower among THA patients (60 years, range 40-75) compared to the other treatment groups $(p<0.001)$. The CCI was significantly higher among patients treated with THA compared to the other treatment groups $(p<0.001)$. Mean follow-up was 40 months, and it did not differ among treatment groups. Patients having a revision due to a failure were 2-6 years younger in their treatment groups. Patient demographics are summarized in Table 1.

The primary outcome of implant survival with revision due to any cause for the different treatment groups was as follows: THA, $85 \%$ at 1 years and $82 \%$ at 2 years; HA, $97 \%$ at 1 and 2 years; SHS, $76 \%$ at 1 and 2 years; and cannulated screws, $53 \%$ at 1 years and $13 \%$ at 2 years. The difference was statistically significant both between THA and HA and SHS and cannulated screws $(p<0.001)$ (Fig. 2). There were no statistically significant differences between the THA and HA groups with respect to the number of nonoperatively treated primary complications $(p=0.1)$ or patient survival (Fig. 3). Approximately 50\% of all femoral neck fractures in male patients $<70$ years old occurred in alcoholics (Fig. 4). 
Fig. 1 Flow chart

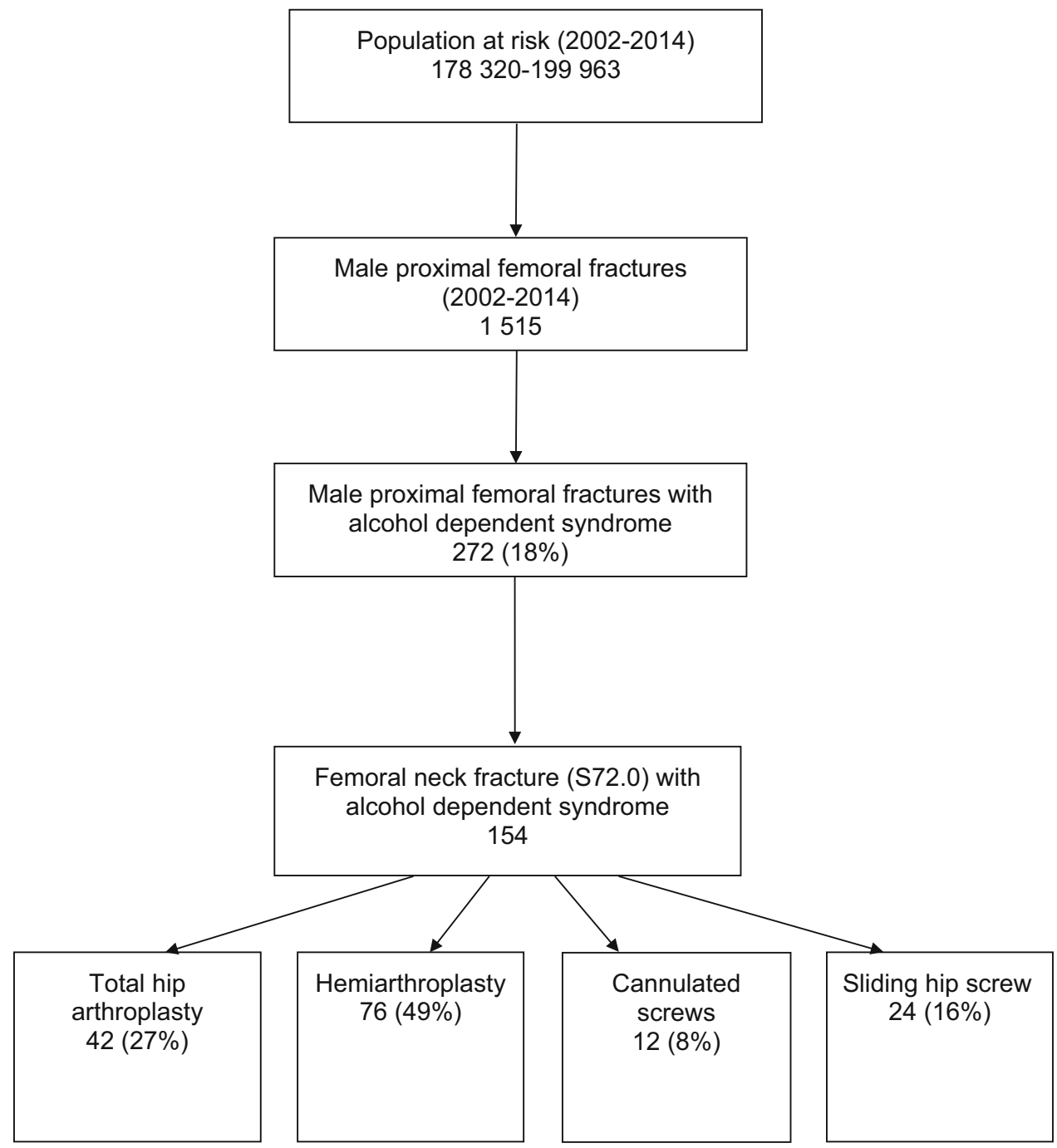

Table 1 Patient demographics

\begin{tabular}{|c|c|c|c|c|c|c|}
\hline & Toal & THA & HA & Sliding hip screw & Cannulated screws & $p$ value \\
\hline Revision & $20(13.5 \%)$ & $9(21 \%)$ & $2(2.7 \%)$ & $3(15 \%)$ & $7(58.3 \%)$ & $<0.001$ \\
\hline Infection & 4 & 2 & 2 & 0 & 0 & \\
\hline Dislocation & 2 & 2 & 0 & 0 & 0 & \\
\hline Periprosthetic fracture & 4 & 5 & 0 & 0 & 0 & \\
\hline Failure & 10 & 0 & 0 & 3 & 7 & \\
\hline Number of revisions & 1.6 & 2.3 & 1.0 & 1.1 & 1.5 & 0.3 \\
\hline CCI median (range) & 2.0 & 2.0 & 4.0 & 2.0 & 2.0 & $<0.001$ \\
\hline Mean age at fracture in years (range) & $64(29-94)$ & $60(40-75)$ & $69(51-94)$ & $61(46-79)$ & $54(29-74)$ & $<0.001$ \\
\hline Mean age for patients with complication (range) & $55(29-71)$ & $58(40-74)$ & $66(60-71)$ & $55(40-65)$ & $47(29-62)$ & \\
\hline Mean follow-up in months (range) & $40(0-150)$ & $41(0-106)$ & $41(0-150)$ & $28(0-82)$ & $55(0-147)$ & 0.5 \\
\hline Total & 149 & $42(28 \%)$ & $76(49 \%)$ & $24(16 \%)$ & $12(8 \%)$ & \\
\hline
\end{tabular}

The overall unadjusted incidence of hip fractures in men was 58.5 per 100,000 person-years [ $95 \%$ confidence interval (CI) 55.6-61.5] for all male hip fractures. The overall unadjusted incidence of male hip fractures with ADS was 9.8 per 100,000 person-years (95\% CI 8.6-11.0). Age-specific incidences in male patients with ADS are shown in Fig. 5. Used THA, type of fixation, and complications related to the used THA are summarized in Table 2. 


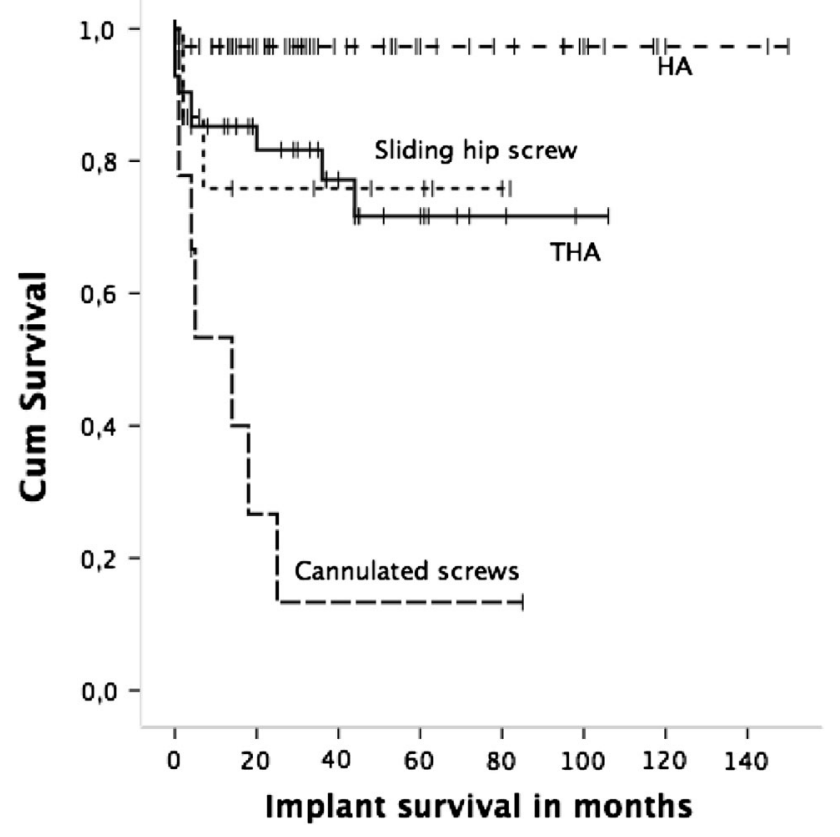

Fig. 2 Implant survival with revision due to any reason stratified by different surgical methods $(p<0.001)$

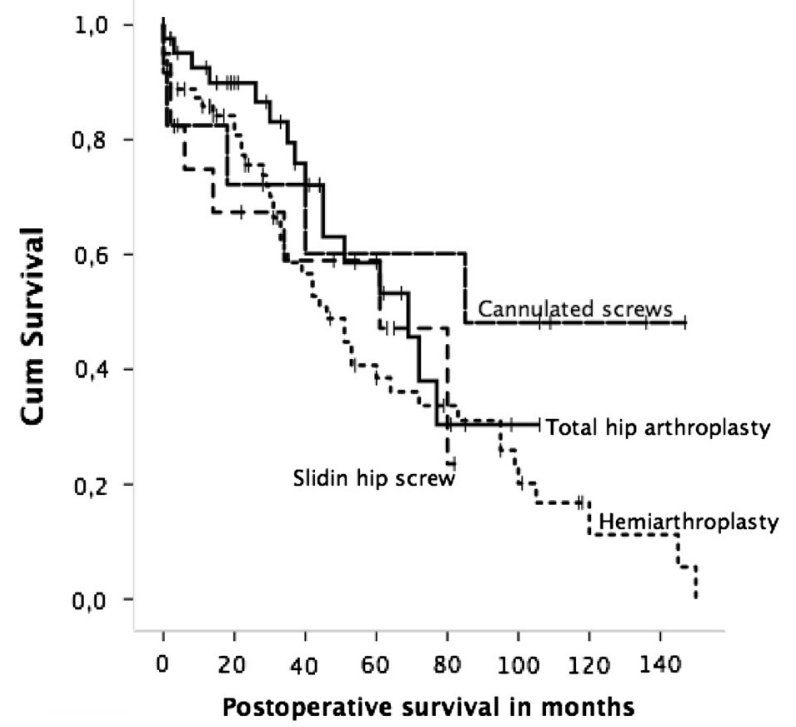

Fig. 3 Patient survival stratified by different surgical methods $(p=0.3)$

\section{Discussion}

Although the overall hip fracture incidence has not changed in the last two decades, in the last 5 years, the incidence has remained stable in women but increased in men [8]. A recent article [9] stated that a small portion of femoral neck fractures in young adults are caused by highenergy trauma, while lifestyle factors and other nontraumarelated risk factors are major contributors. Several authors from different countries have reported high rates of alcohol consumption in younger patients with hip fracture [10], but we have not identified any reports with incidences as high as those in our study. We found a remarkably high incidence of hip fractures in patients of 20-70 years old: half of the patients with a low-energy hip fracture were with ADS.

There is a little evidence regarding the optimal treatment option for alcoholics, and treatment is usually chosen based on expert opinion. We found that there was a substantial difference in implant survival between the treatment groups, with the SHS superior to cannulated screws when internal fixation is used. According to the current literature, the stability of SHS fixation is superior to cannulated screws, particularly in vertical and basal fractures, which are more unstable [11-14]. Our results suggest that stable fixation may be more important in ADS patients, as many trauma patients continue to drink after sustaining an injury and thus may be less likely to follow mobility restrictions during postoperative rehabilitation $[15,16]$.

Displaced femoral neck fractures can be treated by THA or HA for primary replacement of the femoral head. The decision of whether to employ THA or HA should be guided by the patient's age, activity level, and predicted remaining life span. ADS patients tend to be younger than the typical older patient who experiences a hip fracture; in our study, surgeons appeared to prefer THA, presumably for younger ADS patients with better overall health status as measured by the CCI. In addition, in our study, the preoperative physiological status was well established by $\mathrm{CCI}$; however, in young ADS patients, CCI cannot be used to predict patient survival, since death rates were similar among the different treatment groups. Hip fracture ADS patients had increased mortality compared to those who did not abuse alcohol: overall survival was $63 \%$ at 1 year and $49 \%$ at 2 years.

The most striking result of our study was that implant survival with revision due to any reason as the endpoint for THA was significantly worse than for HA. This result was unexpected, as the previous studies have clearly shown the benefits of THA over HA in younger patient groups [17]. Although ADS patients tend to be younger and without impaired mobility, they have a dramatically increased risk of falling. Moreover, the habit of heavy drinking does not subside after the initial hip fracture operation [15]. ADS patients who undergo THA appear to have a higher risk of periprosthetic fracture and dislocation compared to ADS patients treated with HA. The larger femoral head size in $\mathrm{HA}$ is an obvious and well-known factor for improved stability [18]. The same was observed in THA patients as large mega-heads, used at the time, we more stable than THAs with normal head. Not only the risk of revision increased with THA; the number of revisions was also 
Fig. 4 Proportion of patients with ADS within different age groups
Fig. 5 Twelve-year mean incidence (per 100,000 personyears with $95 \%$ confidence intervals) of hip fracture in ADS male population by age group
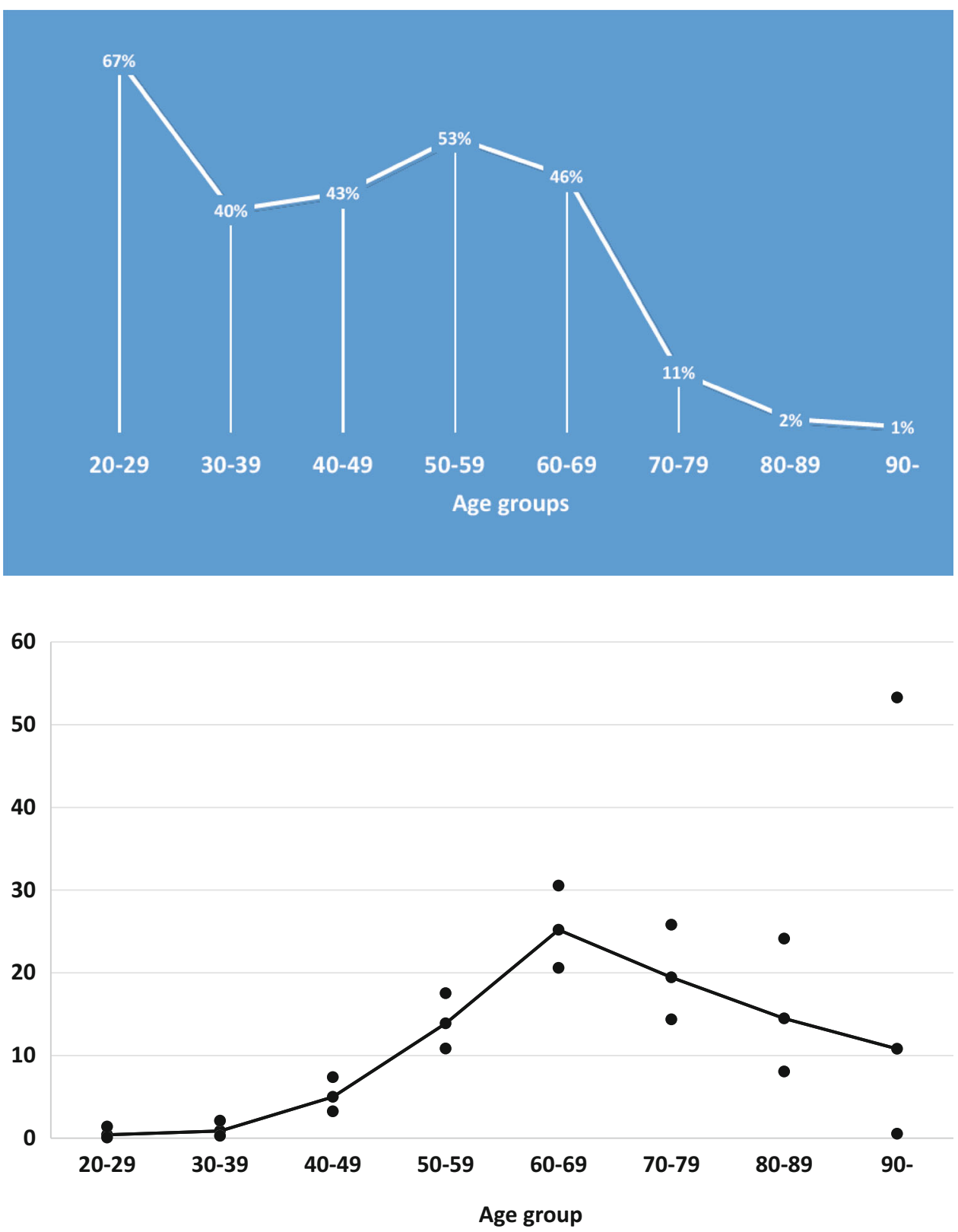

Table 2 Used total hip arthroplasties and complications related to prosthesis used

\begin{tabular}{|c|c|c|c|c|c|c|c|}
\hline Stem & Acetabulum & Head & Dislocation & $\begin{array}{l}\text { Dislocation } \\
\text { requiring } \\
\text { revision }\end{array}$ & $\begin{array}{l}\text { Periprosthetic } \\
\text { fracture }\end{array}$ & Infection & $\begin{array}{l}\text { Complications in } \\
\text { total }\end{array}$ \\
\hline Cementless & Cementless & Large & $0 / 19$ & $0 / 19$ & $2 / 19$ & $1 / 19$ & $3 / 19$ \\
\hline Cementless & Cementless & Normal & $1 / 9$ & $2 / 9$ & $1 / 9$ & $1 / 9$ & $6 / 9$ \\
\hline Cemented & Cemented & Normal & $2 / 13$ & $0 / 13$ & $1 / 13$ & $0 / 13$ & $3 / 13$ \\
\hline Cemented & Cemented & Constrained & $0 / 1$ & $0 / 1$ & $1 / 1$ & $0 / 1$ & $1 / 1$ \\
\hline
\end{tabular}

higher for THA than for HA, although this finding did not reach statistical significance. In the present study, reoperation due to periprosthetic fracture was associated with high rates of postoperative morbidity and mortality, in concordance with the literature. The reported prevalence of periprosthetic fractures after elective THA treatment is 
$0.1-4 \%$, which is far less than the $10 \%$ that we observed; treatment results for these periprosthetic fractures have poor outcomes, a high frequency of complications, and mortality similar to our results [19-21].

This study has several limitations. First, it was retrospective and consequently lacked randomization. Second, although the total number of patients was relatively high, the numbers in different subgroups and the catchment population were small, making statistical analysis of variables difficult. Third, this was a register study and lacked any patient-related outcome measures. A strength of the present work is that we could reliably include all hip fractures in men in a single well-defined population; moreover, all patients' files were available. We were able to gather data for the majority of patients in the region, as our health care system is available to everyone and coverage of injured patients is excellent.

In conclusion, we found that femoral neck fracture patients who were documented to have ADS represented nearly half of patients $<70$ years old with low-energy hip fracture. ADS patients may be more difficult to treat successfully, as they are more likely to have complications after surgery. The stability of internal fixation appeared to play major role in nondisplaced femoral neck fractures, and use of the SHS should be considered over multiple cannulated screws. In displaced fractures, THA carried a significantly higher risk of complications leading to revision compared to HA within this ADS patient group. An advantage of THA over HA is that it may help to avoid revisions due to acetabular wear or pain due to acetabular impingement, although no revisions were performed for these reasons in the patients included in the present study.

Acknowledgements We acknowledge financial support for this study from Competitive Research Funding of Tampere University Hospital.

\section{Compliance with ethical standards}

Conflict of interest The authors declare that they have no conflict of interest.

Ethical approval This article does not contain any studies with human participants or animal performed by any of the authors.

Funding We acknowledge financial support for this study from Competitive Research Funding of Tampere University Hospital.

Informed consent Informed consent was obtained from all individual participants included in the study.

\section{References}

1. Omsland TK, Emaus N, Tell GS, Magnus JH, Ahmed LA, Holvik K, Center J, Forsmo S, Gjesdal CG, Schei B, Vestergaard P, Eisman JA, Falch JA, Tverdal A, Sogaard AJ, Meyer HE (2014) Mortality following the first hip fracture in Norwegian women and men (1999-2008). A NOREPOS study. Bone 63:81-86. doi:10.1016/j.bone.2014.02.016

2. Holmberg AH, Johnell O, Nilsson PM, Nilsson JA, Berglund G, Akesson K (2005) Risk factors for hip fractures in a middle-aged population: a study of 33,000 men and women. Osteoporos Int 16:2185-2194. doi:10.1007/s00198-005-2006-1

3. Benetos IS, Babis GC, Zoubos AB, Benetou V, Soucacos PN (2007) Factors affecting the risk of hip fractures. Injury 38:735-744. doi:10.1016/j.injury.2007.01.001

4. Bikle DD, Genant HK, Cann C, Recker RR, Halloran BP, Strewler GJ (1985) Bone disease in alcohol abuse. Ann Intern Med 103:42-48

5. Kool B, Ameratunga S, Jackson R (2009) The role of alcohol in unintentional falls among young and middle-aged adults: a systematic review of epidemiological studies. Inj Prev 15:341-347. doi:10.1136/ip.2008.021303

6. Zhang X, Yu Z, Yu M, Qu X (2015) Alcohol consumption and hip fracture risk. Osteoporos Int 26:531-542. doi:10.1007/ s00198-014-2879-y

7. Charlson ME, Pompei P, Ales KL, MacKenzie CR (1987) A new method of classifying prognostic comorbidity in longitudinal studies: development and validation. J Chronic Dis 40:373-383

8. van der Velde RY, Wyers CE, Curtis EM, Geusens PP, van den Bergh JP, de Vries F, Cooper C, van Staa TP, Harvey NC (2016) Secular trends in fracture incidence in the UK between 1990 and 2012. Osteoporos Int. doi:10.1007/s00198-016-3650-3

9. Al-Ani AN, Neander G, Samuelsson B, Blomfeldt R, Ekstrom W, Hedstrom M (2013) Risk factors for osteoporosis are common in young and middle-aged patients with femoral neck fractures regardless of trauma mechanism. Acta Orthop 84:54-59. doi:10. 3109/17453674.2013.765639

10. Lofthus CM, Osnes EK, Meyer HE, Kristiansen IS, Nordsletten L, Falch JA (2006) Young patients with hip fracture: a population-based study of bone mass and risk factors for osteoporosis. Osteoporos Int 17:1666-1672. doi:10.1007/s00198-006-0176-0

11. Samsami S, Saberi S, Sadighi S, Rouhi G (2015) Comparison of three fixation methods for femoral neck fracture in young adults: Experimental and Numerical Investigations. J Med Biol Eng 35:566-579. doi:10.1007/s40846-015-0085-9

12. Gardner S, Weaver MJ, Jerabek S, Rodriguez E, Vrahas M, Harris M (2015) Predictors of early failure in young patients with displaced femoral neck fractures. J Orthop 12:75-80. doi:10. 1016/j.jor.2014.01.001

13. Razik F, Alexopoulos AS, El-Osta B, Connolly MJ, Brown A, Hassan S, Ravikumar K (2012) Time to internal fixation of femoral neck fractures in patients under sixty years-does this matter in the development of osteonecrosis of femoral head? Int Orthop 36:2127-2132. doi:10.1007/s00264-012-1619-1

14. Siavashi B, Aalirezaei A, Moosavi M, Golbakhsh MR, Savadkoohi D, Zehtab MJ (2015) A comparative study between multiple cannulated screws and dynamic hip screw for fixation of femoral neck fracture in adults. Int Orthop 39:2069-2071. doi:10. 1007/s00264-015-2881-9

15. Nyquist F, Overgaard A, Duppe H, Obrant KJ (1998) Alcohol abuse and healing complications after cervical hip fractures. Alcohol Alcohol 33:373-380

16. Levy RS, Hebert CK, Munn BG, Barrack RL (1996) Drug and alcohol use in orthopedic trauma patients: a prospective study. J Orthop Trauma 10:21-27

17. Rogmark C, Leonardsson O (2016) Hip arthroplasty for the treatment of displaced fractures of the femoral neck in elderly patients. Bone Jt J 98-B:291-297. doi:10.1302/0301-620X.98B3. 36515

18. Girard J (2015) Femoral head diameter considerations for primary total hip arthroplasty. Orthop Traumatol Surg Res 101:S25S29. doi:10.1016/j.otsr.2014.07.026 
19. Lindahl H, Garellick G, Regner H, Herberts P, Malchau H (2006) Three hundred and twenty-one periprosthetic femoral fractures. J Bone Jt Surg Am 88:1215-1222. doi:10.2106/JBJS.E.00457

20. Meek RM, Norwood T, Smith R, Brenkel IJ, Howie CR (2011) The risk of peri-prosthetic fracture after primary and revision total hip and knee replacement. J Bone Jt Surg Br 93:96-101. doi:10.1302/0301-620X.93B1.25087
21. Sheth NP, Brown NM, Moric M, Berger RA, Della Valle CJ (2013) Operative treatment of early peri-prosthetic femur fractures following primary total hip arthroplasty. J Arthroplasty 28:286-291. doi:10.1016/j.arth.2012.06.003 